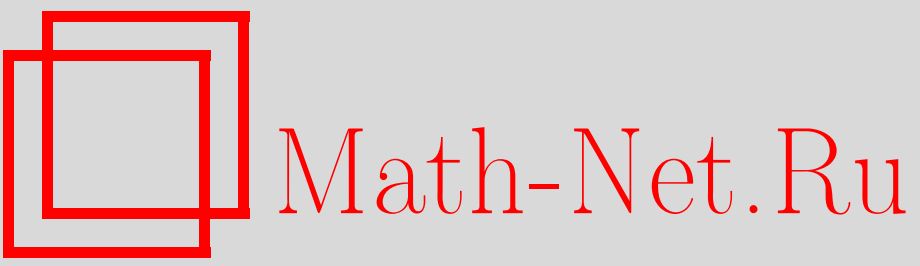

Ф. А. Богомолов, А. Л. Городенцев, В. А. Исковских, Ю. И. Манин, В. В. Никулин, Д. О. Орлов, А. Н. Паршин, В. Я. Пидстригач, А. С. Тихомиров, Н. А. Тюрин, И. Р. Шафаревич, Андрей Николаевич Тюрин (некролог), УМН, 2003, том 58, выпуск 3, 176-182

DOI: https://doi.org/10.4213/rm1631

Использование Общероссийского математического портала Math-Net.Ru подразумевает, что вы прочитали и согласны с пользовательским соглашением

http://www.mathnet.ru/rus/agreement

Параметры загрузки:

IP : 3.81 .55 .215

26 апреля 2023 г., 06:36:41 


\section{АНДРЕЙ НИКОЛАЕВИЧ ТЮРИН}

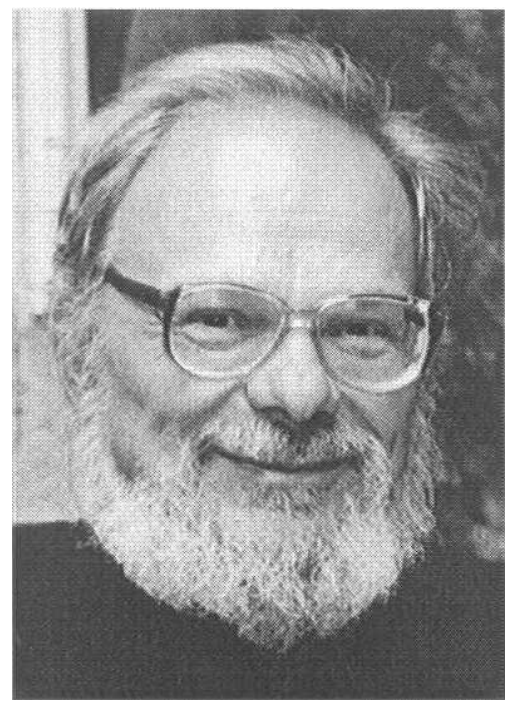

27 октября 2002 г. на 63-м году жизни скоропостижно скончался член-корреспондент Российской академии наук, главньй научньй сотрудник отдела алгебры Математического института им. В.А. Стеклова РАН Андрей Николаевич Тюрин. Выдающийся математик, один из крупнейших специалистов в алгебраической и дифференциальной геометрии, Андрей Николаевич никогда не останавливался на достигнутом - он всегда жил еще не осуществленными планами и новыми идеями, которых у него было множество. Достаточно отметить, что только за последние 10 лет им написано более 25 работ. Широко образованный, интересующийся различными областями не только математики, но и математической физики и квантовой теории поля, Андрей Николаевич до самого последнего дня продолжал активно работать сразу в нескольких направлениях. Смерть пришла неожиданно и мгновенно - не выдержало сердце. Многие математические идеи остались нереализованными, остались незавершенными работы по калибровочньм теориям на графах и по алгебраической лагранжевой геометрии, недописанньми статьи о многообразиях Калаби-Яу и многообразиях Фано. Ушел из жизни человек, глубоко преданный своему делу, человек, искренне влюбленньй в математику, понимающий и тонко чувствующий ее красоту, ученый, смотрящий далеко вперед, открытьй всему новому, исследователь, мыслящий оригинально, находя нестандартные решения сложных задач.

Вся математическая жизнь Андрея Николаевича была связана с Математическим институтом им. В.А. Стеклова РАН, в котором он проработал ровно 40 лет. В 1962 г. он был принят в качестве аспиранта в отдел алгебры и с того времени до самого последнего дня оставался одним из самых активно работающих сотрудников этого отдела - отдела, который был для него родным.

Андрей Николаевич Тюрин родился в 1940 г. в Москвев семье военных инженеров. В 1957 г. он вслед за своей старшей сестрой Галиной (также ставшей впоследствии известным математиком) поступил на механико-математический факультет МГУ, где его научньп руководителем стал Игорь Ростиславович Шафаревич. Его интерес сразу привлекла тогда еще новая область алгебраической геометрии - теория векторных расслоений. С появлением работ А. Вейля, Ж. П. Серра, а затем Д. Мамфорда векторные расслоения вошли в математическую жизнь в качестве мощного геометрического инструмента. Как потом оказалось, эта глубокая и богатая теория стала одной из центральных как в алгебраической геометрии, так и в математической жизни самого Андрея Николаевича.

Уже в первых своих работах [1], [2], [4] А.Н. Тюрин получил сильные результаты: им была решена универсальная задача для квазирасслоений (т.е. расслоений, оснащенных исключительным подрасслоением) на кривой произвольного рода и было доказано, что многообразие модулей 
стабильных векторных расслоений на кривой является изотривиальным расслоением над рациональной базой с рациональным слоем. Эти результаты легли в основу его кандидатской диссертации, которую он защитил в 1965 г.

Продолжая исследование векторных расслоений на кривых, Андрей Николаевич доказал [5], [6], что сама кривая однозначно определяется многообразием модулей стабильных расслоений с фиксированным рангом и первым классом Черна. Теоремы такого рода о восстановлении по некоторьм данным самого многообразия называются теоремами типа Торелли, имея в виду классический результат Торелли о восстановлении кривой по ее якобиану как главнополяризованному абелеву многообразию. Эти результаты А. Н. Тюрина произвели сильное впечатление и сейчас уже стали классическими. Они составили его докторскую диссертацию, которую он защитил в 1971 г.

Теоремы типа Торелли о восстановлении многообразия по некоторым данным всегда играли важную роль в математике. Одной из таких проблем была классическая задача о восстановлении 3-мерной кубики по ее поверхности Фано. Поверхность Фано, параметризующая прямые на кубике, изучалась еще в работах итальянских геометров и, в частности, в работах самого Фано. Эта проблема была блестяще решена А.Н. Тюриным. В работах [7] и [8] Андрей Николаевич показал, что поверхность Фано однозначно определяется своим многообразием Альбанезе (периодами регулярных 1-форм) и сама кубика однозначно восстанавливается по этой поверхности. Можно сказать, что данные статьи предвосхитили первую часть знаменитой работы Клеменса и Гриффитса о нерациональности гладкой 3-мерной кубики.

Эти исследования были завершены яркой обзорной статьей [9], которая оказала существенное влияние на развитие бирациональной теории 3-мерных алгебраических многообразий в последующие годы. В этой работе, кроме очень ясного геометрического изложения теории многообразий Прима применительно к расслоениям на коники, было введено новое понятие, получившее в дальнейшем название многообразия Прима-Тюрина. Оно появилось в связи с изучением промежуточных якобианов 3-мерных многообразий Фано с одномерной группой Пикара. Оказалось, что введенное таким способом главнополяризованное абелево многообразие связано со многими другими геометрическими конструкциями, возникающими в различных смежных областях: в теории интегрируемых систем, в теории представлений алгебр Ли и во всех вопросах, где приходится использовать спектральные кривые.

Еще один блестящий результат, который вырос из классической алгебраической геометрии и сам стал классическим, был получен А. Н. Тюриным в работе [13]. Изучая связки квадрик, Андрей Николаевич доказал, что каждая такая связка однозначно определяется своей кривой вырождения и неэффективной $\vartheta$-характеристикой на ней. Более того, он показал, что, и обратно, любая гладкая кривая на проективной плоскости с неэффективной $\vartheta$-характеристикой получается из некоторой связки квадрик. Эту теорему можно также назвать теоремой типа Торелли.

Работая с пространствами модулей кривых, в начале 70-х годов Андрей Николаевич начал интересоваться нестандартными проективными структурами на римановых поверхностях, т.е. такими структурами, которые не индуцируются с универсального накрытия. Различные проективные структуры, некоторые из которых соответствуют дискретным квазифуксовым группам в $P G L(2, \mathbb{C})$, могут быть получены добавлением квадратичного дифференциала.

Исследуя этот вопрос, Андрей Николаевич построил соответствие, которое проективной структуре и квадратичному мероморфному дифференциалу на римановой поверхности сопоставляет проективно плоское расслоение с сечением [16]. В частности, было показано, что проективно плоское расслоение с сечением фактически определяется главной частью квадратичного дифференциала.

В следующей работе [17] он описал пространство модулей проективно плоских структур на римановой поверхности рода $g>1$ как аффинное расслоение над модулями комплексных структур, где слоем является пространство квадратичных дифференциалов. Фактически это афффинное расслоение ассоциировано с векторньм кокасательным расслоением на пространстве модулей комплексных структур. Основным результатом было явное описание коцикла, определяющего данное аффинное расслоение.

Каждый цикл своих работ Андрей Николаевич завершал хорошей обзорной статьей. Работа [18] - одна из них: в ней излагается теория проективных связностей, квадратичных диффференциалов и их периодов, а также даются геометрические объяснения большинству вычислений, использующих шварцеву производную. 
В 1981 г. Андрей Николаевич нашел замечательную конструкцию, которая дает локальные и глобальные инварианты четырехмерных псевдоримановых многообразий [21], [25]. Они получили название инвариантов Тюрина. Основная идея этой конструкции заключается в следующем. Для каждой точки $x$ четырехмерного псевдориманова многообразия $M$ рассматривается шестимерное векторное пространство $\Lambda^{2} T(x)$, которое является внешним квадратом касательного пространства $T(x)$ в точке $x$. Внешний квадрат метрики $\Lambda^{2} G(x)$, форма объема $\operatorname{Vol}(x)$ и риманова форма кривизны $R(x)$ в точке $x$ определяют три квадратичные формы на пространстве $\Lambda^{2} T(x)$. Проективизация пересечения определяемых ими трех квадрик является поверхностью типа К 3 с естественной вещественной структурой (или некоторое вырождение поверхностей К3). Таким образом, каждой точке $x$ четырехмерного псевдориманова многообразия $M$ каноническим образом сопоставляется некоторая $\mathrm{K} 3$-поверхность $K 3(x)$, и, следовательно, возникает естественное отображение многообразия $M$ в пространство модулей поверхностей К 3 , назьваемое индикатрисой Тюрина. Андрей Николаевич показал, что известные типы метрик такие, как эйнштейнова, автодуальная и другие, соответствуют различным алгебро-геометрическим типам K3-поверхностей и их вырождений. Данная конструкция, которая сразу же вызвала огромньй интерес, существенно уточняет и обобщает известный инвариант Петрова пространств Эйнштейна. Инвариант Тюрина имеет глубокую физическую природу, разбивая точки четырехмерного пространства-времени на различные типы подобно разбиению точек риманова многообразия на точки положительной, отрицательной или нулевой кривизны.

Огромньй вклад внес Андрей Николаевич в теорию многомерных расслоений на алгебраических поверхностях и трехмерном проективном пространстве. Эта область алгебраической геометрии, исключительно глубокая и богатая, имеет многочисленные приложения в топологии и теоретической физике. Андрей Николаевич привнес в теорию векторных расслоений свое исключительно глубокое понимание классической алгебраической геометрии, что позволило ему стать одним из лидеров в этой области.

Используя описание Барта расслоений на $\mathbb{P}^{3}$ в терминах линейной алгебры, Андрей Николаевич в работе [22] дает явную геометрическую конструкцию для математических инстантонов с $c_{2}=4$, показьвая, что каждьй такой инстантон однозначно восстанавливается по кривой вырождения связки квадрик и "неэффективной" $\vartheta$-характеристике (половине канонического класса) на ней. В работах [23], [24] вводится операция суперпозиции инстантонов, которая позволила Андрею Николаевичу доказать унирациональность многообразия модулей инстантонов с зарядом $c_{2}$, равным 4 и 5 .

Одновременно с изучением расслоений на проективных пространствах А.Н.Тюрин получил ряд сильных результатов о многообразиях модулей расслоений ранга 2 на односвязных поверхностях общего типа и поверхностях K3 [27], [37], [37]. В частности, он показал, что многообразия модулей таких расслоений обладают естественной пуассоновой структурой, но не одной, а зависящей от выбора универсального расслоения, что явилось существенным обобщением результатов Мукаи для расслоений на поверхностях К3. Кроме того, им было показано, что модули простых расслоений с фиксированными инвариантами на поверхности К3 бирационально эквивалентны симметрической степени самой поверхности К3. Он также сформулировал ряд гипотез о структуре различных многообразий модулей расслоений на поверхностях, которые во многом определили дальнейшее развитие этой области алгебраической геометрии.

В первой половине 80-х годов геометрия и топология подверглись мощному воздействию идей и методов, пришедших из математической физики. Одним из примеров является применение калибровочной теории в четырехмерной топологии. Особенностью этого направления была его тесная связь с алгебраической геометрией, а именно с теорией векторных расслоений. Теория векторных расслоений долгое время являлась одним из основных методов вычисления инвариантов гладких структур на алгебраических поверхностях, по той причине, что структура стабильного расслоения по существу совпадает с наличием антиавтодуальной связности на расслоении. $\mathrm{K}$ этому же времени относится и серия гипотез о классификации алгебраических поверхностей как гладких четьрехмерных многообразий, самой известной из которых является гипотеза Ван де Вена.

Как непревзойденный знаток векторных расслоений Андрей Николаевич сразу и с особым воодушевлением включился в работу над этими проблемами. В первой статье на эту тему [37] дан обзор существующей на то время техники при работе с модулями инстантонов и полиномами До- 
нальдсона. Особую роль играли наблюдения о влиянии геометрии поверхности на ее гладкие инварианты. Здесь, по-видимому, впервые обсуждается роль канонического класса как инварианта гладкой структуры, роль, ставшая впоследствии ведущей, как показала теория Зайберга-Виттена.

Обладая богатейшим опытом геометрического анализа, А. Н. Тюрин решил предпринять попытку доказать гипотезу Ван де Вена, утверждающую, что поверхности разной кодаировой размерности не могут быть диффеоморфны. В совместной статье с В. Пидстригачем [37] эту гипотезу удалось доказать в важном частном случае: для минимальных поверхностей. В ходе работы оказалось, что алгебро-геометрические вычисления заметно сложнее, чем можно было ожидать, и только изумительная изобретательность Андрея Николаевича позволила успешно завершить данный проект.

В работах [37], [38], [40] А. Н. Тюрин реализовал программу построения спин-полиномиальных инвариантов гладкости. В этих работах, как и в предыдущей, циклы на многообразии модулей, необходимые для определения инвариантов, задавались через условие подскока ядра скрученного с инстантоном оператора Дирака, что в переводе на алгебро-геометрический язык означает подскок размерности пространств когомологий расслоения и, вследствии этого, допускает подробньй геометрический анализ. Этот подход значительно улучшил вьчислимость спин-полиномиальных инвариантов по сравнению с полиномами Дональдсона, позволив использовать так называемьй процесс геометрической аппроксимации пространств модулей. В работах [39], [41], [42] конструируются так называемые канонические спин-полиномы, которые при различии гладких структур оказьваются эффективней всех остальных спин-полиномов. Основная проблема здесь состоит в том, что многообразия модулей оказываются аномально большой размерности. В данных работах было показано, как вычислять спин-полиномы и в такой ситуации. Кроме того, был существенно усовершенствован процесс геометрической аппроксимации.

Ландшафот теории гладких 4-мерных структур радикально изменился с появлением инвариантов Зайберга-Виттена. Неожиданно для многих оказалось, что добавление в систему уравнений из работы [37] нелинейного члена делает многообразия модулей решений и соответствующие инварианты достаточно чуткими к гладкой структуре несмотря на абелевость калибровочной группь. Виттен предложил физические аргументы в пользу того, что новые инварианты эквивалентны полиномам Дональдсона. В работе [44] была предложена программа математического доказательства данного факта.

Как и прежде, завершая свою деятельность в данной области, Андрей Николаевич написал замечательньй обзор [45], оформленньй в виде цикла лекций, в котором основной акцент делается на взаимоотношении деформационных типов и гладких структур на алгебраических поверхносT9X.

В последние годы Андрей Николаевич очень успешно и плодотворно разрабатьвал сразу два направления: алгебраическую лагранжеву геометрию и неабелевы $\vartheta$-функции на римановых поверхностях.

Лагранжева геометрия приобрела особое значение с появлением в математике такого физического феномена, как зеркальная симметрия. В работе [50], изучая компактные гладко погруженные лагранжевы циклы $S \subset M$ фиксированного топологического типа в данном симплектическом многообразии $M$, Андрей Николаевич показал, что фиксация на $M$ почти комплексной структуры, согласованной с симплектической формой, позволяет для каждого лагранжева подмногообразия $S \subset M$ определить фазу Маслова $m: S \rightarrow S^{1}$. Циклы, на которых она постоянна, называются специальными лагранжевыми . Специальные лагранжевы циклы составляют в бесконечномерном многообразии всех лагранжевых циклов $\mathfrak{L}$ подмногообразие конечной размерности, равной $\operatorname{dim} H^{1}(S)$, и являются специальным случаем косоголоморфных циклов, отвечающим критическому (равному $\pi / 2$ ) значению кэлерова угла. Основным содержанием этой работы является попытка соединить зеркальные - лагранжеву и голоморфную (с кэлеровым углом 0) геометрии непрерьвным семейством "косоголоморфных" геометрий и показать, что традиционные геометрические проблемы могут исследоваться средствами, в равной мере приложимыми к любому значению кэлерова угла.

С другой стороны, дополнительная фиксация на $M$ данных геометрического предквантования выделяет в $\mathfrak{L}$ подмногообразие бор-зоммерфельдовых циклов, которое имеет конечную коразмерность, также равную $\operatorname{dim} H^{1}(S)$. Вопрос о его расположении по отношению к многооб- 
разию специальных лагранжевых циклов представлялся А. Н. Тюрину крайне важным. В [50], [47], [51] исследуются пересечения подмногообразия бор-зоммерфельдовых циклов со специальными $\operatorname{dim} H^{1}(S)$-мерньми семействами лагранжевых циклов, возникающими при наличии на $M$ комплексной или вещественной поляризации. В обоих случаях конечное число точек пересечения соответствует естественным специальным базисам в голоморфных линейных системах, в пространствах $\vartheta$-функций, в конформных блоках и т. п. В статьях [48], [55] показано, что если на $M$ зафиксировать $M p^{\mathbb{C}}$-структуру ${ }^{1}$, то эта структура индуцирует на лагранжевых циклах полувеса (внешние формы "полустаршей степени"), и многообразие полувзвешенных бор-зоммерфельдовых циклов фиксированного объема снабжается канонической интегрируемой кэлеровой структурой и голоморфным линейным расслоением, оснащённым голоморфиными данными геометрического предквантования.

Другое направление деятельности А.Н. Тюрина в последние годы связано с изучением пространства модулей $\Re$ классов эквивалентных представлений $\pi_{1}(\Sigma) \rightarrow S U(2)$ фундаментальной группы римановой поверхности $\Sigma$ рода $g$. Это продолжение еще аспирантской деятельности А. Н. Тюрина, относящейся к изучению симплектической геометрии многообразия $\mathfrak{R}$. Разрезание $\Sigma$ на стандартные 3 -валентные штаны по $3 g-3$ петлям определяет на многообразии $\mathfrak{R}$ симплектическое действие $(3 g-3)$-мерного тора: каждая нетривиальная петля $C \subset \Sigma$ задает на $\mathfrak{R}$ функцию Голдмана, сопоставляющую представлению $\varrho$ число $\pi^{-1} \arccos (\operatorname{tr}(\varrho(C)) / 2)$, и гамильтоново векторное поле этой функции определяет на $\mathfrak{R}$ действие окружности $U(1)$. Отображение моментов относительно этого действия расслаивает многообразие $\mathfrak{R}$ на симплектические торические орбиты над выпуклым многогранником $\Delta_{\Gamma}$, который называется многогранником Дельцана. Комбинаторика данного многогранника описывает примькание торических орбит.

Статьи [52], [57], [59] посвящены явному комбинаторному описанию $\mathfrak{R}$ как симплекто-торического многообразия. В частности, в работе [59] дано описание топологии $\mathfrak{R}$, не апеллирующее к комплексной геометрии, откуда сразу следует независимость разных характеристик $\Re$ от выбора комплексной структуры на кривой. В статье [57] доказана гипотеза Флорентино: построено явное взаимно однозначное и гладкое во внутренних точках отождествление многогранника Дельцана с унитарным пространством Шоттки $u S_{g}$, которое является фактором прямого произведения $g$ экземпляров $S U(2)$ по диагональному действию $S U(2)$.

Трудно описать должным образом все научные достижения А. Н. Тюрина, еще труднее оценить то влияние, которое он оказал на математиков, особенно молодых, кто так или иначе общался с ним. Андрей Николаевич имел большое число учеников и последователей. Неформальный в разговоре, живо интересующийся мнением собеседника он щедро делился своими мыслями и идеями. Его семинар, проходивший в Математическом институте РАН на протяжении многих лет, пользовался огромной популярностью. Жизнелюбивьй, влюбленньй в математику, Андрей Николаевич заряжал своей энергией всех окружающих.

Живой и открытый в общении, он имел огромное количество друзей по всему миру. Ему нравилось встречать новых людей, ему было интересно жииь и работать.

Ф.А. Богомолов, А. Л. Городенцев, В.А. Исковских, Ю. И. Манин, В. В. Никулин, Д. О. Орлов, А. Н. Паршин, В. Я. Пидстригач, А. С. Тихомиров, Н. А. Тюрин, И. Р. Шафаревич

\section{СПИСОК НАУЧНЫХ РАБОТ А.Н. ТЮРИНА}

[1] О классификации двумерных векторных расслоений над алгебраической кривой произвольного рода // Изв. АН СССР. Сер. матем. 1964. Т. 28. № 1. С. 21-54

[2] Классификация векторных расслоений над алгебраической кривой произвольного рода // Изв. АН СССР. Сер. матем. 1965. Т. 29. № 3. С. 657-688

[3] Алгебраические поверхности // Труды МИАН. 1965. Т. 75

[4] О классификации $n$-мерных векторных расслоений над алгебраической кривой произвольного рода // Изв. АН СССР. Сер. матем. 1966. Т. 30. № 6. С. 1353-1366

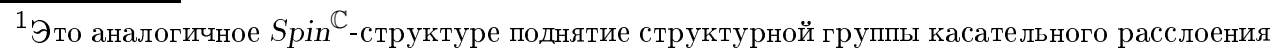
на $M$ с симплектической до $U(1)$-расширения ее двулистной накрьвающей. 
[5] Аналог теоремы Торелли для двумерных расслоений над алгебраической кривой произвольного рода // Изв. АН СССР. Сер. матем. 1969. Т. 33. № 5. С. 1149-1170

[6] Аналог теоремы Торелли для многомерных векторных расслоений над произвольной алгебраической кривой // Изв. АН СССР. Сер. матем. 1970. Т. 34. № 2. С. 338-365

[7] О поверхности Фано неособой кубики в $P^{4} / /$ Изв. АН СССР. Сер. матем. 1970. Т. 34. № 6. C. $1200-1208$

[8] Геометрия поверхности Фано неособой кубики $F \in P^{4}$ и теоремы Торелли для поверхностей Фано и кубики // Изв. АН СССР. Сер. матем. 1971. Т. 35. № 3. С. 498-529

[9] Пять лекций о трехмерных многообразиях // УМН. 1972. Т. 27. № 5. С. 3-50

[10] О множестве особенностей дивизора Пуанкаре многообразия Пикара поверхности Фано неособой кубики // Изв. АН СССР. Сер. матем. 1972. Т. 36. № 5. С. 947-956

[11] Геометрия модулей векторных расслоений // УМН. 1974. Т. 29. № 6. С. 59-88

[12] О пересечении квадрик // УМН. 1975. Т. 30. № 6. С. 51-99

[13] Об инварианте связки квадрик // Изв. АН СССР. Сер. матем. 1975. Т. 39. № 1. С. 23-27

[14] Геометрия дивизора Пуанкаре многообразия Прима // Изв. АН СССР. Сер. матем. 1975. T. 39. № 5. C. 1003-1043

[15] Конечномерные расслоения на бесконечных многообразиях // Изв. АН СССР. Сер. матем. 1976. T. 40. № 6. C. $1248-1268$

[16] Периоды и главные части квадратичных дифференциалов на оснащенной римановой поверхности // Изв. АН СССР. Сер. матем. 1977. Т. 41. №6. С. 1425-1442

[17] Обобщение ю-функций Вейерштрасса и многообразие модулей оснащенных римановых поверхностей // Изв. АН СССР. Сер. матем. 1978. Т. 42. № 5. С. 1151-1161

[18] О периодах квадратичных дифференциалов // УМН. 1978. Т. 33. №6. С. 149-195

[19] Средний якобиан трехмерных многообразий // Итоги науки и техники. Совр. проблемы матем. Т. 12. М.: ВИНИТИ, 1979. С. 5-57

[20] Геометрия особенностей общей квадратичной формы // Изв. АН СССР. Сер. матем. 1980. T. 44. № 5. C. 1200-1211

[21] Локальный инвариант риманова многообразия // Изв. АН СССР. Сер. матем. 1981. Т. 45. № 4. C. $824-851$

[22] Структура многообразия пар коммутирующих пучков симметрических матриц // Изв. АН CССР. Сер. матем. 1982. Т. 46. № 2. С. 409-430

[23] On the superpositions of mathematical instantons // Progr. Math. 1983. V. 36. P. 433-450

[24] The instanton equations for $(n+1)$-superpositions of marked $a d T P^{3} \oplus \operatorname{adTP} P^{3} / / \mathrm{J}$. Reine Angew. Math. 1983. V. 341. P. 131-146

[25] Локальньй и глобальный инварианты четырехмерного псевдориманова многообразия // Труды МИАН. 1984. Т. 165. С. 205-219

[26] Исследования по геометрии алгебраических многообразий в отделе алгебры МИАН // Труды МИАН. 1985. Т. 168. С. 98-109

[27] Специальные 0-циклы на поляризованной поверхности типа $K 3$ // Изв. АН СССР. Сер. матем. 1987. Т. 51. № 1. С. 131-151

[28] Инстантонная пленка для $\left(\mathbb{C P}^{2}, \mathrm{can}\right) / /$ Доклады по математике и ее приложениям. Т. 1. № 1. Москва-Тула: МИАН СССР-Тульский политехнический институт, 1987. С. 123-147

[29] Cycles, curves and vector bundles on an algebraic surface // Duke Math. J. 1987. V. 54. P. 1-26

[30] Симплектические структуры на многообразии модулей векторных расслоений на алгебраических поверхностях с $p_{g}>0$ // Изв. АН СССР. Сер. матем. 1988. Т. 52. № 4. С. 813-851

[31] Алгебро-геометрические аспекты гладкости. 1. Полиномы Дональдсона // УМН. 1989. T. 44. № 3. C. $93-143$

[32] A slight generalization of the Mehta-Ramanathan theorem // Algebraic Geometry (Proceedings of the US-USSR Symposium, Chicago, IL, 1989). Berlin: Springer-Verlag, 1989. P. 258-272. (Lecture Notes in Math. V. 1479.)

[33] The geometry of the special components of moduli space of vector bundles over algebraic surfaces of general type // Complex Algebraic Varieties (Proc. Conf., Bayreuth, 1990). Berlin: Springer-Verlag, 1990. P. 166-175. (Lecture Notes in Math. V. 1507.)

[34] The moduli spaces of vector bundles on threefolds, surfaces and curves I // Erlangen, 1990

[35] Метрика Вейля-Петерсона на пространстве модулей стабильных векторных расслоений и пучков над алгебраической поверхностью // Изв. АН СССР. Сер. матем. 1991. Т. 55. № 3. C. $600-630$ 
[36] Инварианты гладкой структуры алгебраической поверхности, задаваемые оператором Дирака // Изв. РАН. Сер. матем. 1992. Т. 56. № 2. С. 279-371 (совм. с В.Я. Пидстригачем)

[37] A simple method of distinguishing the underlying differentiable structures of algebraic surfaces // Preprint. Universität Göttingen. Mathematica Gottingensis, Sonderforschungsbereichs "Geometry and Analysis". Heft 25, 1992

[38] Спин-полиномиальные инварианты гладких структур на алгебраических поверхностях // Изв. РАН. Сер. матем. 1993. Т. 57. № 2. С. 125-164

[39] Almost canonical polynomials of algebraic surfaces // Algebraic Geometry and its Applications (Proc. Conf., Yaroslavl, 1992 / ed. A. Tikhomirov, A. Tyurin. Braunschweig: Vieweg, 1993. P. 227-251. (Aspects Math. V. E 25.)

[40] Canonical and almost canonical spin polynomials of an algebraic surface // Vector Bundles in Algebraic Geometry (Proceedings of the Symposium, Durham, England, 1993 / ed. N. J. Hitchin et al. Cambridge: Cambridge Univ. Press, 1995. P. 255-281. (London Math. Soc. Lecture Note Ser. V. 208.)

[41] Spin canonical invariants of 4-manifolds and algebraic surfaces // Warwick Preprints № 38/1994. P. 1-23

[42] Канонические спинполиномы алгебраической поверхности. 1 // Изв. РАН. Сер. матем. 1994. T. 58. № 6. C. 157-204

[43] Application of geometric approximation procedure to computing the Donaldson polynomials for $\mathbb{C P}^{2} / /$ Preprint. Universität Göttingen. Mathematica Göttingensis, Sonderforschungsbereichs "Geometry and Analysis". Heft 12, 1994. P. 1-71 (with A. Tikhomirov)

[44] Localisation of the Donaldson's Invariants along Seiberg-Witten Classes // Preprint 95-075. Bielefeld: Univ. Bielefeld, Sonderforschungsbereich 343 "Diskrete Structuren in der Mathematik", 1995 (with V. Pidstrigach)

[45] Six lectures on four-manifolds // Lecture Notes in Math. 1996. V. 1646. P. 186-246

[46] The classical geometry of vector bundles // Algebraic Geometry (Ankara, 1995). New York: Dekker, 1997. P. 347-378. (Lecture Notes in Pure and Appl. Math. V. 193.)

[47] Quantization and "theta function" // Preprint № 216. Paris: Université Paris VI-VII, 1999; math.AG/9904046

[48] Complexification of Bohr-Sommerfeld conditions // Preprint № 15. Oslo: Inst. of Math., Univ. Oslo, 1999

[49] Geometric quantization and mirror symmetry // Preprint № 22. Warwick, 1999; math.AG/ 9902027

[50] Специальная лагранжева геометрия как малая деформация алгебраической геометрии (GQP и зеркальная симметрия) // Изв. РАН. Сер. матем. 2000. Т. 64. № 2. С. 141-224

[51] О базисах Бора-Зоммерфельда // Изв. РАН. Сер. матем. 2000. Т. 64. № 5. С. 163-196

[52] Three mathematical faces of SU(2)-spin networks // Preprint № 35/2000. Lisboa: IST, 2000; math.DG/0011035

[53] Analytical aspects of the theory of non abelian theta functions // Preprint. Lisboa: IST, 2000 (with C. Florentino, J. Mourao, J. Nunes)

[54] Неабелевы аналоги теоремы Абеля // Изв. РАН. Сер. матем. 2001. Т. 65. № 1. С. 123-180

[55] Абелева лагранжева алгебраическая геометрия // Изв. РАН. Сер. матем. 2001. Т. 65. № 3. С. 15-50 (совм. с А. Л. Городенцевым)

[56] Quantization, classical and quantum field theory and theta-functions // 2002; math.AG/ 0210466

[57] Решеточные калибровочные теории и гипотеза Флорентино / / Изв. РАН. Сер. матем. 2002. T. 66. № 2. C. 205-224

[58] On the Mumford-Narasimhan problem // Preprint № 129. Bonn: Max-Planck-Institute für Mathematik, 2002

[59] Модели Дельцана пространств модулей // Изв. РАН. Сер. матем. 2003. Т. 67. № 2. С. 167-180

[60] Fano versus Calabi-Yau // Proceedings of the Fano Conference, Turin, Italy, 2002 (to appear)

[61] Предисловие редактора перевода // Четырехмерная риманова геометрия (семинар Артура Бессе 1978/79). М.: Мир, 1985. С. 5-6

[62] От редактора перевода $/ / \Phi$. Клейн. Лекции об икосаэдре и решении уравнений пятой степени. М.: Наука, 1989. С. 3-8 